\title{
A Vehicle-to-Microgrid (V2M) Framework with Optimization-Incorporated Distributed EV Coordination for a Commercial Neighborhood
}

\author{
Md Shamiur Rahman, Member, IEEE, M. J. Hossain, Senior Member, IEEE, Junwei Lu, Senior Member, IEEE, \\ Fida Hasan Md Rafi, and Sukumar Mishra, Senior Member, IEEE
}

\begin{abstract}
An improved vehicle-to-microgrid (V2M) framework is proposed for a commercial locality operating as a hybrid alternating-current (AC) / direct-current (DC) microgrid, which optimally coordinates electric-vehicle (EV) storages in a distributed manner. An aggregator model is proposed that solves the economic dispatch problem of parked EV storages in a centralized fashion and generates power references in real-time for the designed $\mathrm{EV}$ storage controllers. Unlike the conventional EV storage controller, the proposed optimizationincorporated distributed EV storage controller (ODC) can switch from decentralized to distributed control mode or vice versa based on situations and utilizes a sparse vehicle-to-vehicle (V2V) communication network. The power flow between the $\mathrm{AC}$ and DC subgrids is managed by interlinking converters (IC). The IC control structure is augmented by combining voltage- and powerbased droop-control schemes in the power control loop. This modification enables simultaneous $\mathrm{AC}$ and $\mathrm{DC}$ bus voltage regulations. Case studies are carried out to validate the efficacy of the developed framework with a real commercial network and loads. The results exhibit robust performance of the overall system for generation-demand variability, transitions between islanded and grid-tied conditions, and user-preferred EV disconnections and time delay.
\end{abstract}

Index Terms-Hybrid AC/DC microgrid, economic dispatch, interlinking inverter, electric vehicles, distributed cooperative control.

\section{INTRODUCTION}

$\mathbf{T}$ HE concept of a direct-current (DC) power distribution network is gaining momentum due to the increased number of photovoltaic (PV) installations along with the rapid growth in electric vehicles (EV) as promising energy storage systems (ESS). The DC distribution system can provide a cost-effective solution to integrate DC technologies such as light-emitting diodes (LED), DC motors, data centers etc. with minimum conversion losses. Commercial buildings, that consume nearly $61 \%$ of the total electric energy in the United States, are emerging as zero-net-energy (ZNE) buildings by adopting DC distribution systems [1]. As a result, islanded DC distribution systems or inter-tied DC microgrids are possible by expanding the capacities of rooftop PV and ESS in

Md Shamiur Rahman and M. J. Hossain are with the School of Engineering, Macquarie University, NSW 2109, Australia (e-mail: mdshamiur.rahman@students.mq.edu.au; jahangir.hossain@mq.edu.au).

Junwei Lu is with the Griffith School of Engineering, Griffith University, Gold Coast, QLD 4222, Australia (e-mail: j.lu@griffith.edu.au).

Fida Hasan Md Rafi is with the ElectraNet Pty Ltd., Adelaide, S. A. 5000, Australia (e-mail: fida.rafi@students.mq.edu.au).

Sukumar Mishra is with the Indian Institute of Technology Delhi, India (e-mail: sukumar@ee.iitd.ac.in). commercial buildings. However, the installation of a DC-only distribution system is unlikely in the near future due to the ACdominated legacy electric system. Therefore, an intermediate solution would be hybrid AC/DC microgrids [2], [3]. Hybrid microgrids combine the aptness of both AC and DC systems. They facilitate the connection of both the AC and DC types of loads and sources due to the existence of individual buses of different nature. Moreover, there are other advantages such as minimum AC/DC conversion loss, increased efficiency, and reduced voltage synchronization effort, to ensure improved connectivity for AC and DC technologies [4], [5]. However, this microgrid structure has convoluted network and control topologies. Due to the intricate nature of their structures and control methodologies, hybrid microgrids are well suited for commercial neighborhood rather than residential localities.

Contemporary commercial buildings are equipped with state-of-the-art technologies such as smart EV parks, intelligent power-electronic-interfaced variable-speed drives (VSDs), four-quadrant inverters and smart high-voltage airconditioning (HVAC) systems. Utilizing these technologies, commercial buildings can contribute to both electric power grids and the electricity market by providing ancillary services [6]. However, coordination among multiple technologies such as EV storages is a difficult task, particularly when these buildings are operating as autonomous microgrids. Furthermore, peak load for commercial areas occurs during mid-daytime; therefore, parked EV storages in commercial neighborhoods can be utilized to provide ancillary services during office hours, which typically range between 9:00 am to 6:30 pm [7], [8]. Considering the above-mentioned possibilities of vehicleto-microgrid (V2M) (i. e. islanded operation) and vehicleto-grid (V2G) (i. e. grid-tied operation) application in commercial vicinities, several pieces of literature have explored this research area. In [9], an economic analysis is presented to show the feasibility of using EV storages in commercialbuilding microgrids. An energy-management system (EMS) for a building with PV units and EV storages is presented in [10], designed to minimize the operating cost considering different charging and discharging profiles. A real-time peak-shaving model to reduce high peak demand combining demand response and load control is presented in [11]. A four-stage optimization algorithm is proposed in [12] for a $\mathrm{PV}$-assisted EV charging station in a commercial building to minimize operation costs associated with customer satisfaction by the optimal scheduling of EV storages. However, all the 
optimization approaches to coordinate EV storages for V2M operations follows either the hourly or the daily forecasting methods. As a result, these approaches are only suitable for the planning purposes of the V2M. Conversely, a dynamic framework to ensure EV coordination in conjunction with the real-time optimization technique can provide both technical and economic optimality.

The V2M connection can be achieved by centralized, decentralized or distributed approaches for AC, DC or hybrid AC/DC microgrids. The centralized structure provides improved controllability with reduced scalability. The decentralized structure ensures modularity, however, optimal operation cannot be guaranteed due to the limited information exchange among units. Conversely, a distributed structure combines the advantages of both centralized and decentralized structures. As a result, distributed control in microgrids with $\mathrm{EV}$ storages is gaining momentum. Distributed controllers are developed for AC microgrids [13], [14], for DC microgrids [15]-[17] and for hybrid AC/DC microgrids [8], [18]. The application of distributed control for ESS is presented in [19]-[23] to achieve voltage control, state-of-charge balancing and power-sharing considering time delays and uncertainties in PV output power generation. However, these analyses do not specifically concentrate on commercial-grade V2M applications, and the concept of incorporating a solution for the EV economic dispatch problem in their real-time control has not been explored. Some recent work [24], [25], has attempted to unify optimizationbased economic dispatch with real-time distributed control for DC microgrids. However, their feasibility and efficacy for hybrid-microgrid operations are not explored. Moreover, from a realistic point of a view, an EV aggregator would like to have a certain control over the EV storages under its operational vicinity, but at the same time, EV storages are parked in a spatially dispersed form. Therefore, from the perspective of an EV aggregator and EV owners, a combination of the centralized and distributed control framework would be an optimal approach for commercial-grade V2M operation.

Addressing all the above-mentioned issues, this paper presents a novel V2M framework suitable for commercial neighborhoods. The hybrid AC/DC microgrid structure is adopted due to its suitability for commercial operation. An improved control framework is proposed to integrate $\mathrm{EV}$ aggregators, which involves three core components: (i) a centralized EV economic-dispatch optimizer, (ii) a distributed EV storage controller and (ii) an enhanced interlinking converter (IC) with modified control topology. The optimizer generates the reference power for each EV storage as a function of the incremental cost $(\lambda)$ by solving an economic dispatch problem. Later, it adjusts the power reference accordingly, which ensures proper power management within the microgrid through a cost-effective and distributed V2M operation. The $\mathrm{IC}$ is responsible to control the power flow between the $\mathrm{AC}$ and DC subgrids. The IC control topology is augmented by combining the voltage-based droop [26], [27] and power-based droop-control techniques. This augmentation enables simultaneous AC and DC bus voltage regulation without affecting the power-sharing capabilities of the converter. Overall, the salient features of this paper can be summarized as follows:
1) An aggregator model is developed that provides a centralized solution for the EV economic dispatch problem considering a bounded EV output power. The aggregator/ EMS generates a reference power signal for the designed distributed controller. A generalized mathematical expression of the optimal reference power is presented as a function of the optimal incremental cost, defined by the slope of the cost function. This augmentation ensures that EV storages will operate economically providing maximum profit for EV owners.

2) A distributed EV storage controller is designed that can operate in both decentralized and distributed manner based on a situation-aware signal. The designed controller communicates with other neighboring EV units through a sparse communication network with a spanning tree to exchange output-power information. Later, it utilizes a local voting protocol for dynamic averaging.

3) In order to ensure voltage regulation and to maintain power flow between the AC and DC subgrids, a modified IC control strategy combining power- and voltagebased droop techniques is developed. This enhancement ensures simultaneous AC and DC bus voltage regulations for the hybrid microgrid. Moreover, the transient performance of the IC is further enhanced by utilizing a proportional-derivative (PD) controller within the powercontrol loop of the IC as proposed in [8]. This approach facilitates a smooth transition between islanded and gridtied microgrid operation.

The remainder of the paper is organized as follows: Section II presents the concept and configuration of a typical commercial hybrid AC/DC microgrid. Section III presents the proposed V2M control framework, which includes a description of the optimizer operating as a centralized EV aggregator, the distributed EV storage controller and the IC controller. Case studies are presented in Section IV, for a commercial hybrid $\mathrm{AC} / \mathrm{DC}$ microgrid with four $\mathrm{EV}$ storages operating under one aggregator, for various scenarios. Moreover, a concluding discussion based on the results is provided in this section. Section V concludes the paper.

\section{COMmERCIAl HYBRID AC/DC MICROGRID}

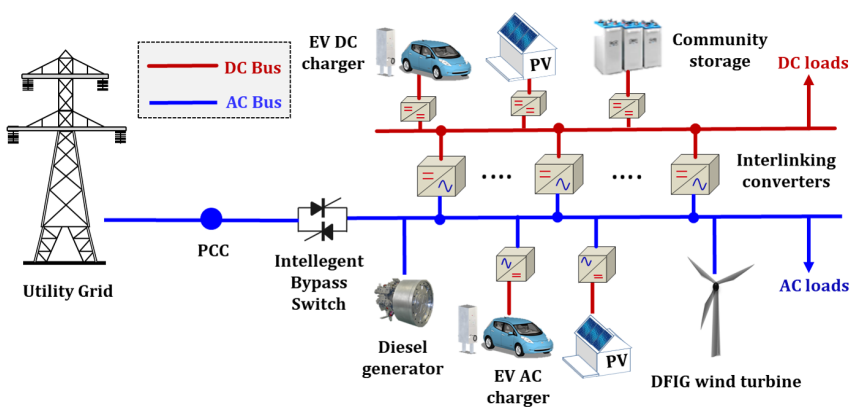

Fig. 1: A general commercial hybrid AC/DC microgrid.

A general configuration of a commercial hybrid AC/DC microgrid is illustrated in Fig. 1. All AC-type sources such as diesel generators, EV AC chargers, doubly-fed induction generators (DFIG), etc. along with AC-type loads are connected to 
the AC bus forming the AC subsystem. Similarly, all DC-type sources such as community storages, EV DC fast chargers, etc. along with all DC-type loads are connected to the DC bus forming the DC subsystem. Both AC and DC subsystem are interfaced through ICs ensuring balanced power flow between two subsystems. The AC subsystem is also connected to the utility grid through intelligent bypass switch (IBS). This configuration enables the microgrid to operate in both grid-tied and islanded operations. Each of the generating components has local controllers to regulate their output power. All of these local controllers are controlled by an energy management system (EMS) which can operate either in a centralized or distributed manner utilizing neighbor-to-neighbor information sharing. The EMS also sends the signal to the IBS to initiate grid reconnection or islanding as per system requirements. The proposed V2M control framework presented in later sections describe how the EMS/aggregator is optimizing the EV output power by interacting with the local controllers of EV storage to ensure stable system operations in both islanded and gridtied scenarios through V2M and V2G modes respectively.

\section{PROPOSED V2M CONTROL FRAMEWORK}

\section{A. EV Aggregator Problem Formulation}

During V2M operation within a hybrid AC/DC microgrid, EV units operate as dispatchable sources. With a view to minimizing the generation cost of EV units while preserving all other flexibilities, the optimization problem can be formulated as similar to that of a conventional power system [24], [28], [29]. Now let us consider that the aggregated cost function, $C(p, t)$ of $N \mathrm{EV}$ storages operating in $\mathrm{V} 2 \mathrm{M}$ mode is a quadratic function of their corresponding output powers with coefficients of $\alpha, \beta$ and $\gamma$. Therefore, the optimization problem for $\mathrm{EV}$ economic dispatch can be presented as follows [24]:

$$
\begin{array}{ll}
\min & C(p, t)=\sum_{n \in N} \alpha_{n}+\beta_{n} p_{n}+\gamma_{n} p_{n}^{2} \\
\text { s.t. } & G=\sum_{n \in N} p_{n}=P_{\text {total }}^{\text {load }}-P_{\text {total }}^{\text {gen }}, n=1,2, \ldots, N . \\
& H_{1}=-p_{n}+p_{n}^{\text {min }} \leq 0 \\
& H_{2}=p_{n}-p_{n}^{\text {max }} \leq 0
\end{array}
$$

where, inequalities $H_{1}$ and $H_{2}$ are $2 \times N$ constraints, $P_{\text {total }}^{\text {load }}=\sum S_{A C}^{\text {load }}+\sum P_{D C}^{\text {load }}$, $P_{\text {total }}^{\text {gen }}=\sum S_{A C}^{\text {gen }}+\sum P_{D C}^{\text {gen }}$,

$\sum S_{A C}^{\text {load }}=$ the aggregated demand of the apparent power by AC loads,

$\sum P_{D C}^{\text {load }}=$ the aggregated demand of the active power by DC loads,

$\sum S_{A C}^{g e n}=$ the aggregated apparent power generated by dispatchable AC sources,

$\sum P_{D C}^{g e n}=$ the aggregated active power generated by nondispatchable DC sources,

$p_{n}^{\max }$ and $p_{n}^{\min }=$ the allowable maximum and minimum power of the $n$th EV storage respectively.

Therefore, the economic-dispatch problem can be formulated as a Lagrange function with Lagrange $(\lambda)$ and
Karush-Kuhn-Tucker (KKT) multipliers $\left(\mu_{1}\right.$ and $\mu_{2}$ which are vectors of $R^{N}$ ) for each EV storage:

$$
L\left(p_{n}, \lambda, \mu_{1}, \mu_{2}\right)=C(p, t)+\lambda G+\mu_{1} H_{1}+\mu_{2} H_{2}
$$

The solution of the aforementioned optimization problem is a stationary point where the partial derivative of $L\left(p_{n}, \lambda\right)$ is zero with respect to all variables. Now, for the time being, if we neglect the EV output power constraints $H_{1}$ and $H_{2}$ then

$$
\begin{aligned}
\frac{\partial L}{\partial p_{n}} & =\frac{\partial C(p, t)}{\partial p_{n}}-\lambda=0 \\
\Longrightarrow \lambda & =\frac{\partial C(p, t)}{\partial p_{n}}=\beta_{n}+2 \gamma_{n} p_{n}
\end{aligned}
$$

In order to achieve optimality, it is necessary that the Lagrange multiplier or the incremental cost $(\lambda)$ of each EV storage converges to the optimal incremental cost $\left(\lambda^{*}\right)$. This convergence can be achieved only when all EV storages generate the optimal power $\left(p_{n}^{*}\right)$. Considering small perturbations of both AC and DC load demands, the optimal output power of each EV storage can be obtained with simple mathematical manipulation of (3), as below [30]-[32]

$$
\left\{\begin{array}{l}
\lambda^{*}=\frac{\Delta \mathcal{P}+\sum_{n \in N} \frac{\beta_{n}}{2 \gamma_{n}}}{\sum_{n \in N} \frac{1}{2 \gamma_{n}}} \\
p_{n}^{*}=\frac{\lambda^{*}-\beta_{n}}{2 \gamma_{n}}
\end{array}\right.
$$

where, $\Delta \mathcal{P}=P_{\text {total }}^{\text {gen }}-P_{\text {total }}^{\text {load }}$. Moreover, by considering the output power constraints, the necessary minimum-cost operating condition can be extended to represent the reference power for the distrubuted EV storage controller.

$$
p_{n}^{r e f}=\left\{\begin{aligned}
p_{n}^{*} & \text { for } p_{n}^{\text {min }} \leq p_{n} \leq p_{n}^{\max } \\
p_{n}^{\text {min }} & \text { for } \quad p_{n} \leq p_{n}^{\text {min }} \\
p_{n}^{\text {max }} & \text { for } \quad p_{n} \geq p_{n}^{\text {max }}
\end{aligned}\right.
$$

\section{B. Optimization-Incorporated Distributed Control (ODC) for EV Storages}

Let us consider a commercial negihborhood operating as a hybrid microgrid with $N$ V2M-capable EV storages. Additionally, all active EV storages communicate through a sparse communication network with each other. The interaction among active EV storages can be represented via a weighted directed graph (digraph). The interconnection nodes can be represented by $\mathcal{G}=\left(\mathcal{V}_{\mathcal{G}}, \mathcal{E}_{\mathcal{G}}, \mathcal{A}_{\mathcal{G}}\right)$, where $\mathcal{V}_{\mathcal{G}}=$ $\left\{\mathcal{V}_{1}, \mathcal{V}_{2}, \mathcal{V}_{3}, \ldots . \mathcal{V}_{n}\right\}$ is the set of all nodes, $\mathcal{E}_{\mathcal{G}} \subset \mathcal{V}_{\mathcal{G}} \times \mathcal{V}_{\mathcal{G}}$ is the set of pairs of nodes also known as edges, and $\mathcal{A}_{\mathcal{G}}=\left[a_{i j}\right]_{N \times N}$ is the weighted adjacency matrix of dimension $N \times N$ that gives information regarding the interconnectivity of nodes. The communication between node $i$ and node $j$ can be presented as follows:

$$
a_{i \times j}=\left\{\begin{array}{cc}
1 & \text { if } \\
0 & \text { otherwise }
\end{array} \quad(i, j) \in \mathcal{E}_{\mathcal{G}}\right.
$$

The in-degree matrix is defined as $D_{G}^{i n}=\operatorname{diag}\left\{d_{i}^{i n}\right\}$, i.e. the diagonal matrix of $d_{i}^{i n}=\sum_{j=0}^{n} a_{i j}$. Likewise the out-degree matrix is defined as $D_{G}^{\text {out }}=\operatorname{diag}\left\{d_{i}^{\text {out }}\right\}$, i.e. the diagonal matrix of $d_{i}^{\text {out }}=\sum_{i=0}^{n} a_{j i}$. The Laplacian matrix $\mathcal{L}_{\mathcal{G}}$, defined 


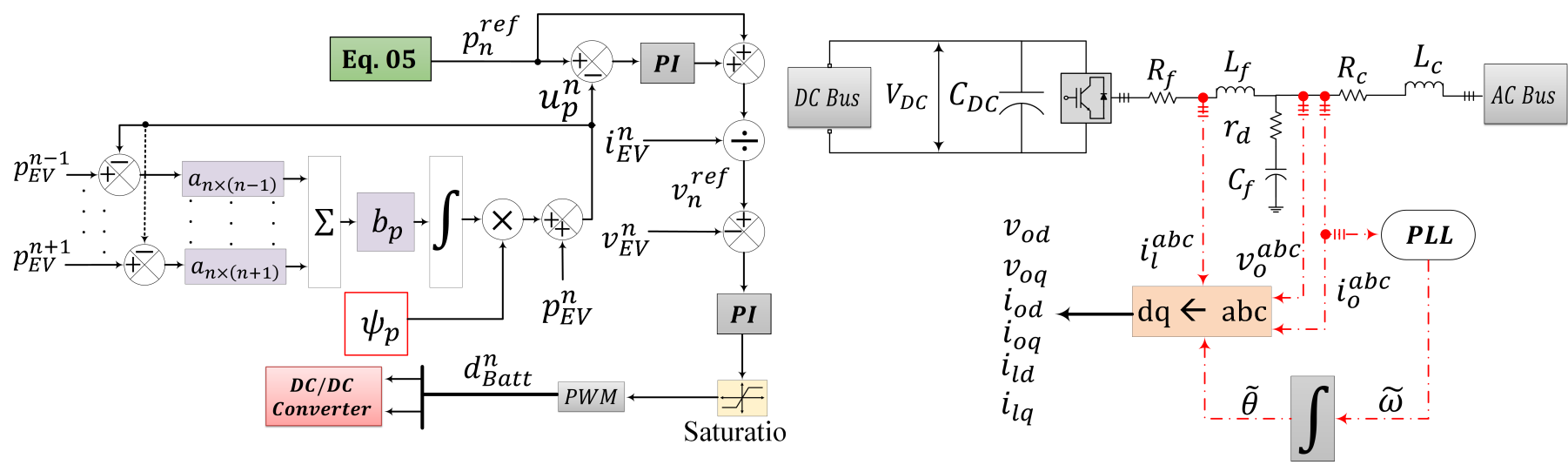

(a)

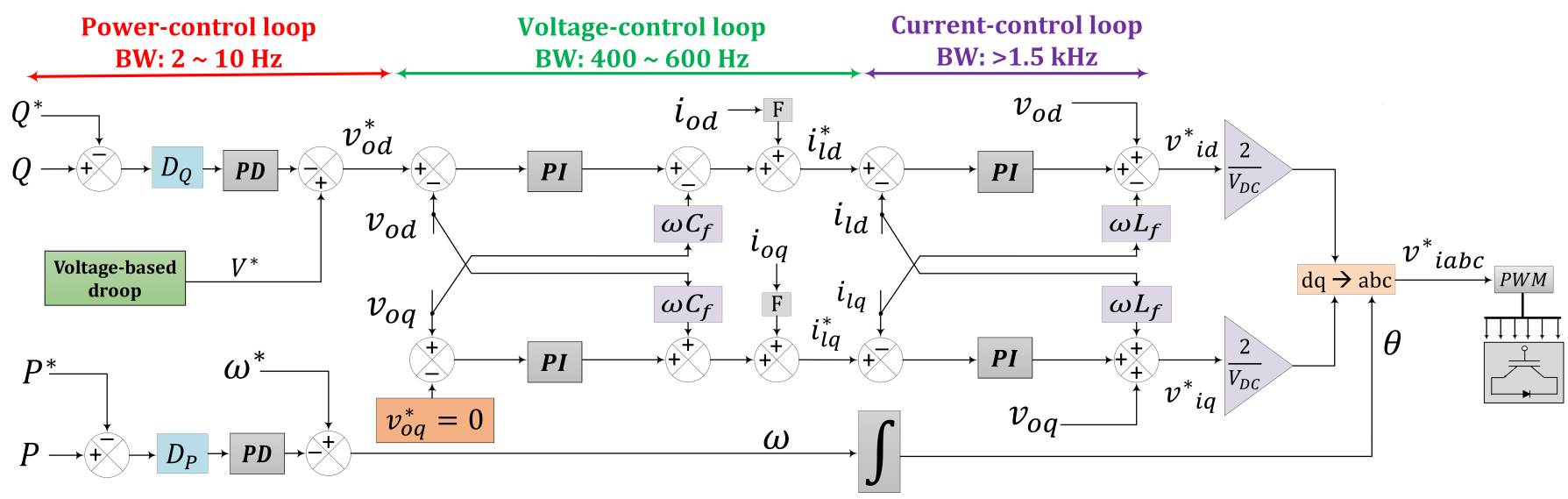

(c)

Fig. 2: Proposed control topology. (a) ODC for individual EV storage.(b) Single-line circuit diagram of each IC. (c) IC control structure with voltage-based droop.

as $\mathcal{L}_{\mathcal{G}}=D_{G}^{i n}-\mathcal{A}_{\mathcal{G}}$, provides useful insights regarding the stability of the communication network. For an undirected communication network with bidirectional information flow, $D_{G}=D_{G}^{o u t}=D_{G}^{i n}$, which results in $\mathcal{L}_{\mathcal{G}}$ being balanced for this particular case [33].

All EV storages are designed with the local voting protocol shown in Fig. 2a. This protocol uses local information of the $n$th EV storage and the information observed from its neighbors to converge to an average output power $u_{p}^{n}$, given by

$$
u_{p}^{n}(t)=p_{E V}^{n}(t)+\psi_{p} b_{p} \int_{0}^{t} \sum_{j \in N} a_{n \times j}\left(p_{E V}^{j}(\tau)-u_{p}^{n}(\tau)\right) d \tau
$$

where $p_{E V}^{n}=$ measured output power of $n^{t h} \mathrm{EV}$ storage, $\psi_{p}=$ acknowledgement signal for the distributed operation which is governed by the need-based distributed coordination strategy (NDCS) [8], $b_{p}=$ coupling gain of the cooperative control loop.

Even though the aggregator generates necessary dispatch command for individual EV storages, the distributed EV storage controller is the part that is monitoring and controlling the EV charging and discharging by considering EV related constraints. The controller is governed by the NDCS algorithm which takes three EV related constraints into account, i.e. storage capacity based on state-of-charge (SOC), converter capacity, and user preference.

The local voting protocol ensures that the output power of all EV storages for any step changes will converge at an average value of the neighboring units at $t \rightarrow \infty$, considering a spanning tree is present and the communication delay $\left(T_{d}\right)$ satisfies the condition [34]

$$
T_{d} \leq \frac{\pi}{2 \max \left(\operatorname{eig}\left(\mathcal{L}_{\mathcal{G}}\right)\right)}
$$

Now by introducing an uniform time delay of $T_{d}$ in (7), the following relation can be obtained

$$
\begin{aligned}
u_{p}^{n}(t) & =p_{E V}^{n}(t) \\
& +\psi_{p} b_{p} \int_{0}^{t} e^{-T_{d} \tau} \sum_{j \in N} a_{n \times j}\left(p_{E V}^{j}(\tau)-u_{p}^{n}(\tau)\right) d \tau
\end{aligned}
$$

Example: let us consider that the output powers of four EV storages for any instant are $20 \mathrm{~kW}, 15 \mathrm{~kW}, 25 \mathrm{~kW}$ and $30 \mathrm{~kW}$ - represented by EV-1, EV-2, EV-3 and EV-4 respectively. A ring-structured communication network is used in this paper. 
(a) $\mathrm{T}_{\mathrm{d}}=0 \mathrm{~s} \quad-\mathrm{EV}-1-\mathrm{EV}-2-\mathrm{EV}-3-\mathrm{EV}-4$

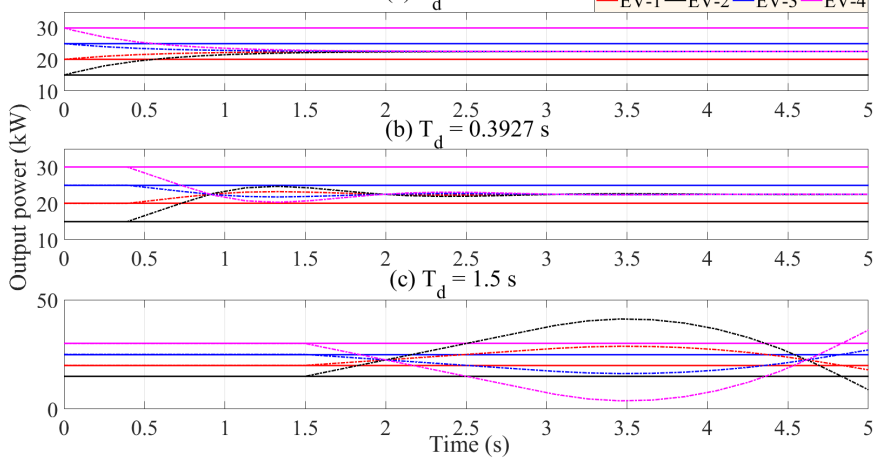

Fig. 3: Convergence of output powers with the distributed controller for various time delays. (a) Time delay, $T_{d}=0 \mathrm{~s}$. (b) Time delay, $T_{d}=0.3927 \mathrm{~s}$ (c) Time delay, $T_{d}=1.5 \mathrm{~s}$. The dotted lines are the outputs measured from the distributed controller.

This network can be mathematically presented as follows:

$$
\begin{aligned}
\mathcal{A}_{\mathcal{G}}= & {\left[\begin{array}{cccc}
0 & 1 & 0 & 1 \\
1 & 0 & 1 & 0 \\
0 & 1 & 0 & 1 \\
1 & 0 & 1 & 0
\end{array}\right] ; \mathcal{D}_{\mathcal{G}}=\left[\begin{array}{cccc}
2 & 0 & 0 & 0 \\
0 & 2 & 0 & 0 \\
0 & 0 & 2 & 0 \\
0 & 0 & 0 & 2
\end{array}\right] ; } \\
\mathcal{L}_{\mathcal{G}}= & {\left[\begin{array}{cccc}
2 & -1 & 0 & -1 \\
-1 & 2 & -1 & 0 \\
0 & -1 & 2 & -1 \\
-1 & 0 & -1 & 2
\end{array}\right] }
\end{aligned}
$$

Therefore, the output power of all EV storages will converge to an average consensus value of $22.5 \mathrm{~kW}$ following (7) as long as the time delay $T_{d}$ is less than $0.3927 \mathrm{~s}$, calculated using (8) The results of this example are presented in Fig. 3, where Figs. $3 \mathrm{a}, 3 \mathrm{~b}$ and $3 \mathrm{c}$ represents the performance of the distributed controller for $T_{d}=0 \mathrm{~s}, 0.3927 \mathrm{~s}$ and $1.5 \mathrm{~s}$ respectively. By differentiating (7) and taking the Laplace transformation with zero initial conditions, we can obtain a generalized model for the cooperative power controller:

$$
\mathbf{U}_{\mathbf{p}}=s\left(s \mathbf{I}_{N \times N}+G_{T D} \psi_{p} b_{p} \mathcal{L}_{\mathcal{G}}\right)^{-1} \mathbf{P}_{\mathbf{E V}}
$$

where the cooperative control output, $\mathbf{U}_{\mathbf{p}}=\left[\begin{array}{lll}u_{p}^{1} & u_{p}^{2} \ldots u_{p}^{n}\end{array}\right]^{T}$, the measured output powers of EV storages, $\mathbf{P}_{\mathbf{E V}}=$ $\operatorname{diag}\left\{p_{E V}^{n}\right\}$, and $G_{T D}=\frac{1}{1+T_{d} s} . \mathbf{I}_{N \times N}$ represents an identity matrix of dimension $N \times N$. Overall, the general formulation of the voltage references, $\mathbf{V}^{\text {ref }}=\left[v_{1}^{r e f} v_{2}^{r e f} \ldots v_{n}^{r e f}\right]^{T}$ for the optimization-incorporated distributed EV storage controller can be expressed as

$$
\mathbf{V}^{\text {ref }}=\left[\mathbf{P}^{\text {ref }}\left(\mathbf{I}_{N \times N}+\mathbf{G}_{\mathbf{p i}(\mathbf{P})}^{\mathbf{E V}}\right)-\mathbf{U}_{\mathbf{p}} \mathbf{G}_{\mathbf{p i}(\mathbf{P})}^{\mathbf{E V}}\right] \mathbf{I}_{\mathbf{E V}}^{-\mathbf{1}}
$$

where $\mathbf{G}_{\mathbf{p i}(\mathbf{P})}^{\mathbf{E V}}=\operatorname{diag}\left\{G_{p i(P)}^{E V}\right\}, \mathbf{P}^{\text {ref }}=\operatorname{diag}\left\{p_{n}^{r e f}\right\}$ and $\mathbf{I}_{\mathbf{E V}}=$ $\operatorname{diag}\left\{i_{E V}^{n}\right\}$ considering $G_{p i(P)}^{E V}$ to be the transfer function of the proportional-integral (PI) controller in the power-control loop of the ODC and $i_{E V}^{n}$ is the output current of the $n$th EV storage.

Overall, the design procedure of the proposed ODC can be summarized as below:

Step 1: From smart meters collect load and generation data then check output powers of active EV storages.
Step 2: Find values of $\alpha, \beta$ and $\gamma$ for all EV storages.

Step 3: Calculate $p_{n}^{r e f}$ using (5).

Step 4: Calculate $\mathbf{V}^{\text {ref }}$ using (11).

Step 5: Repeat the process until $\left|p_{n}^{r e f}-p_{E V}^{n}\right| \approx 0$.

\section{Power-Flow Control between Subgrids via IC}

The objective of the IC is to ensure accurate active and reactive power flow between the $\mathrm{AC}$ and $\mathrm{DC}$ subgrids to maintain the bus voltages and the frequency stability. The single-line circuit diagram of the IC is shown in Fig. 2b. The IC controller consists of three control loops, i.e. power, voltage and current-control loops with bandwidths (BW) of 2-10 Hz, $400-600 \mathrm{~Hz}$ and $1.5 \mathrm{kHz}$ or above respectively as illustrate in Fig. 2c. The voltage and current controllers are designed utilizing a conventional decoupled topology with voltage and current feed-forward techniques, whereas the power-control loop is extended with the combined voltage- and power-based droop techniques proposed in [8]. This augmentation improves concurrent $\mathrm{DC}$ and $\mathrm{AC}$ bus voltage regulation and coordination among AC and DC subgrids. The algebraic expression of the inverter voltage references in $d-q$ reference frame $\left(v_{i d}^{*}\right.$ and $v_{i q}^{*}$ ) and the input angle $\theta$ for the $d-q$ to $a b c$ converter can be presented as below

$$
\left\{\begin{aligned}
v_{i d}^{*} & =v_{o d}-\omega L_{f} i_{l q}+\left[\left\{V^{*}-D_{Q}\left(Q-Q^{*}\right) G_{p d}-v_{o d}\right\} G_{p i}^{V}\right. \\
& \left.-\omega C_{f} v_{o q}+F i_{o d}-i_{l d}\right] G_{p i}^{I} \\
v_{i q}^{*} & =v_{o q}+\omega L_{f} i_{l d}+\left\{\omega C_{f} v_{o d}+\left(v_{o q}^{*}-v_{o q}\right) G_{p i}^{V}\right. \\
& \left.+F i_{o q}-i_{l q}\right\} G_{p i}^{I} \\
\theta & =\frac{1}{s}\left\{\omega^{*}-D_{P}\left(P-P^{*}\right) G_{p d}\right\}
\end{aligned}\right.
$$

where $P$ and $Q$ are the filtered output active and reactive power whereas $P^{*}$ and $Q^{*}$ are the respective references. $v_{l d q}$ and $v_{o d q}$ are the inverter filter input and output voltages, $i_{l d q}$ and $v_{o d q}$ are the inverter filter input and output currents in the $d-q$ reference frame. $D_{P}$ and $D_{Q}$ are active and reactive power droop coefficients. $V^{*}$ is the output of the voltage-based droop-control loop [8] and $F$ is the feed-forward gain. $\omega$ is the angular frequency measured from the phase-locked loop (PLL). $L_{f}$ and $C_{f}$ are the inductance and capacitance values of the LC filter. $G_{p d}, G_{p i}^{V}$ and $G_{p i}^{I}$ are the transfer functions of the PD controller of the power-control loop, the PI controllers of the voltage and current-control loops respectively. Considering $V_{D C}$ to be the measured DC bus voltage, finally, the pulsewidth modulation (PWM) generator of the IC generates the modulation index in the natural $a b c$ reference frame as follows:

$$
\mathbf{M}_{\mathbf{a b c}}=\frac{2}{V_{D C}} \mathbf{T}_{\mathbf{d q o}-\mathbf{a b c}}(\theta) \mathbf{V}_{\mathbf{i d q}}^{*}
$$

where $\mathbf{M}_{\mathbf{a b c}}=\left[\begin{array}{lll}m_{a} & m_{b} & m_{c}\end{array}\right]^{T}, \mathbf{V}_{\mathbf{i d q}}^{*}=\left[\begin{array}{ll}v_{i d}^{*} & v_{i q}^{*}\end{array}\right]^{T}$ and $\mathbf{T}_{\mathbf{d q o}-\mathbf{a b c}}(\theta)$ is the $d q-a b c$ transformation matrix.

\section{CASE STUdiES}

The designed commercial hybrid AC/DC microgrid is illustrated in Fig. 4, and consists of four V2M-capable EV storages parked at the parking area with individual capacities of 24 $\mathrm{kWh}$, one rooftop solar PV unit with a rated capacity of 30 


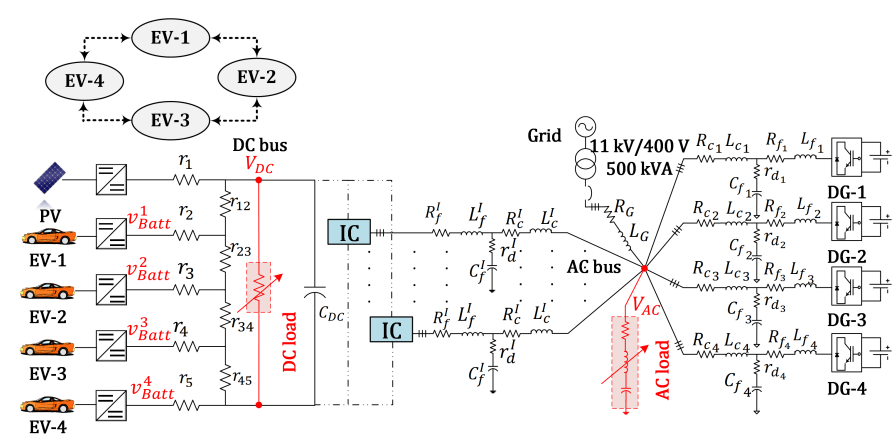

Fig. 4: Single-line circuit diagram of the test hybrid AC/DC microgrid.

$\mathrm{kW}$ and four $17 \mathrm{kVA}$ dispatchable-power electronic-based $\mathrm{AC}$ sources. EV storage models are realistically recalibrated to the specification of Mitsubishi Outlander storage model. The microgrid model presented here is the extended and modified version of the university-based microgrid presented in [7], [8]. All the EV storages and the PV unit are connected to the DC bus of the microgrid. The PV unit is designed to provide maximum power, whereas the EV storages are equipped with the designed ODC. The dispatchable AC sources are connected to the $\mathrm{AC}$ bus via four-quadrant inverters, which are controlled by decentralized droop control scheme as presented in [35]. It should be noted that due to large-value inductors present at the inverter terminals, commercial localities usually have a higher $X / R$ ratio. Thus, decentralized $P-f$ and $Q-V$ droopcontrol schemes are adopted. Both the AC and DC buses are interfaced through a bidirectional interlinking converter, which is controlled by the combined voltage- and power-based droopcontrol scheme.

Three case studies are carried out to demonstrate the efficacy of the proposed V2M framework. The first case study demonstrates the voltage-regulation and power-sharing capabilities of the proposed scheme considering irradiation and load variability. The second case study provides insights on the performance of the framework during and subsequent to grid reconnection. The final case study is performed to show the effects of a sudden V2M service disruption due to the disconnection of particular EV storages. This case study also explores the effects of time delay on the output powers of the EV storages. Parameters relevant to the design of the system are depicted in Table I. The load and solar irradiation data are obtained using OSI PI software which gathers sensor data from the smart meters installed at the university premises.

\section{A. Case A: Variable Solar Insolation and Commercial Load- ing}

For Case A, the designed V2M framework is tested considering PV generation and commercial load variability. The main objective of this case study is to assess the robustness of the developed framework. Real-time irradiation profile for the solar farm and the commercial load data are obtained using the OSI PI software installed for monitoring smart meter data for the Griffith University microrgrid model [7], [8]. Concurrent PV power and load variabilities ensure genuine conditions that the controller needs to withstand. EV storages
TABLE I: System Parameters

\begin{tabular}{|c|c|c|c|}
\hline \multicolumn{4}{|c|}{ Energy nodes } \\
\hline $\begin{array}{l}\text { PV } \\
\text { EV-(1,2,3,4) }\end{array}$ & $\begin{array}{l}30 \mathrm{~kW} \\
24 \mathrm{kWh}\end{array}$ & DG-(1,2,3,4) & $17 \mathrm{kVA}$ \\
\hline \multicolumn{4}{|c|}{ Line parameters } \\
\hline Nominal $V_{A C}^{R M S}$ & $240 \mathrm{~V}$ & $L_{f}$ & $20 \times 10^{-3} \mathrm{H}$ \\
\hline$V_{D C}^{*}$ & $600 \mathrm{~V}$ & $C_{f}$ & $200 \times 10^{-6} \mathrm{~F}$ \\
\hline Frequency & $50 \mathrm{~Hz}$ & $R_{c}, R_{G}$ & $0.03 \Omega$ \\
\hline$C_{D C}$ & $130 \times 10^{-4} \mathrm{~F}$ & $L_{c}, L_{G}$ & $3.5 \times 10^{-4} \mathrm{H}$ \\
\hline$R_{f}^{I}$ & $0.1 \Omega$ & $r_{d}$ & $0.65 \Omega$ \\
\hline $\begin{array}{l}r_{1}, r_{2} \\
r_{3}, r_{4}\end{array}$ & $0.02 \Omega$ & $\begin{array}{l}r_{12}, r_{23} \\
r_{34}, r_{45}\end{array}$ & $0.05 \Omega$ \\
\hline \multicolumn{4}{|c|}{ Control parameters } \\
\hline \multicolumn{2}{|l|}{ Power controller } & \multicolumn{2}{|c|}{ Voltage controller } \\
\hline$\omega_{c}, \omega_{e}$ & $31.42,314.16 \mathrm{rad} / \mathrm{s}$ & $\mathrm{F}$ & 0.19 \\
\hline$\omega^{*}$ & $314.16 \mathrm{rad} / \mathrm{s}$ & $K_{p}$ & 0.11 \\
\hline$D_{P}$ & $3.14 \times 10^{-4}$ & $K_{i}$ & 2.2 \\
\hline$D_{Q}$ & $1.36 \times 10^{-3}$ & \multicolumn{2}{|c|}{ Current controller } \\
\hline$K_{p}$ & $2 \times 10^{-5}$ & $K_{p}$ & 0.16 \\
\hline$K_{d}$ & $2 \times 10^{-7}$ & $K_{i}$ & 200 \\
\hline \multicolumn{4}{|c|}{ EV storage controller } \\
\hline \multicolumn{2}{|c|}{ Power control loop } & \multicolumn{2}{|c|}{ Voltage control loop } \\
\hline$K_{p}$ & 1.3 & $K_{p}$ & 0.03 \\
\hline$K_{i}$ & 6 & $K_{i}$ & 1.1 \\
\hline$b_{p}$ & 1.5 & & \\
\hline \multicolumn{4}{|c|}{ Cost functions of EV storages } \\
\hline EV-1 & \multirow{4}{*}{\multicolumn{3}{|c|}{$\begin{array}{c}120+0.80 p_{1}+0.017 p_{1}^{2} \\
80+0.65 p_{2}+0.012 p_{2}^{2} \\
90+0.60 p_{3}+0.010 p_{3}^{2} \\
100+0.90 p_{4}+0.018 p_{4}^{2}\end{array}$}} \\
\hline EV-2 & & & \\
\hline EV-3 & & & \\
\hline EV-4 & & & \\
\hline
\end{tabular}

communicate through a vehicle-to-vehicle (V2V) communication network illustrated in Fig. 4. As was mentioned before, the designed distributed controller can provide stable operation as long as there exists a spanning tree and the time delay is within a specified limit. The interaction graph among the four EV storages shown in Fig. 4. has one spanning tree. The optimal incremental cost $(\mathrm{c} / \mathrm{kW})$ and the dynamic cost for all EV storages for this case are presented in Figs. 5a and $5 \mathrm{c}$ respectively. It can be observed from the dynamiccost profiles of all EV storages that EV-1 and EV-4 require higher incentives to provide V2M operation than EV-2 and EV-3. Therefore, from Fig. 5e, it can be observed that the EV storages with higher dynamic costs discharge less power for V2M operation. As a result, the highest power is discharged by EV-3 and then EV-2 descending from EV-1 towards EV4. Figs. $5 \mathrm{~b}, 5 \mathrm{~d}$ and $5 \mathrm{f}$ represent electrical parameters such as the AC bus voltage, the normalized DC bus voltage and the system frequency respectively. It can be concluded from the results that the designed V2M framework can provide stable and efficient hybrid microgrid operations in a cost-effective manner.

\section{B. Case B: Effects of Grid Reconnection}

Case B is carried out to demonstrate the efficacy of the designed system during and subsequent to grid reconnection. Microgrids can operate in both islanded and grid-tied modes. 


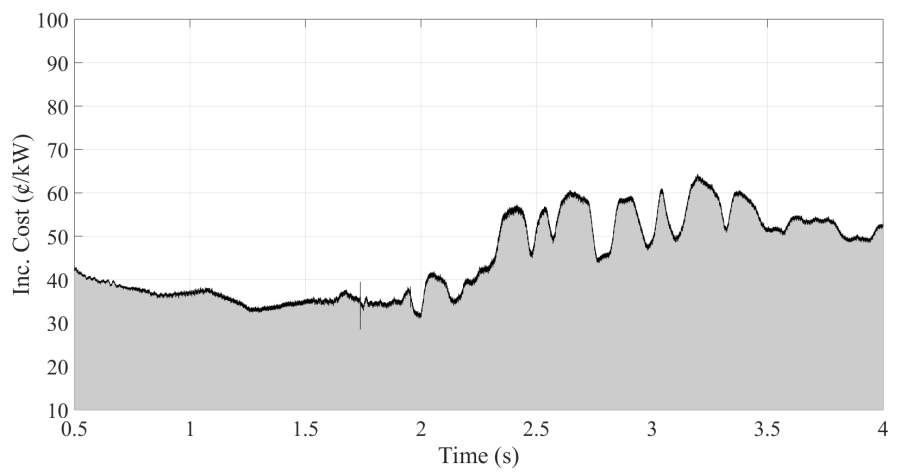

(a)

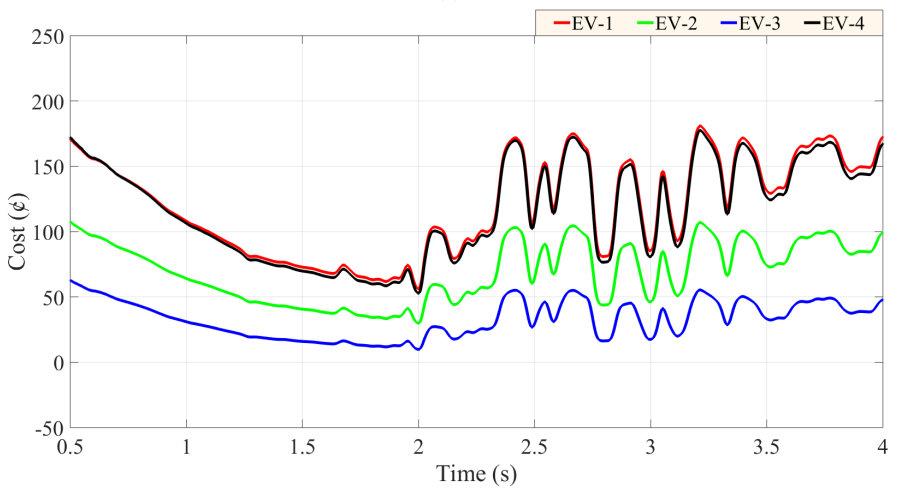

(c)

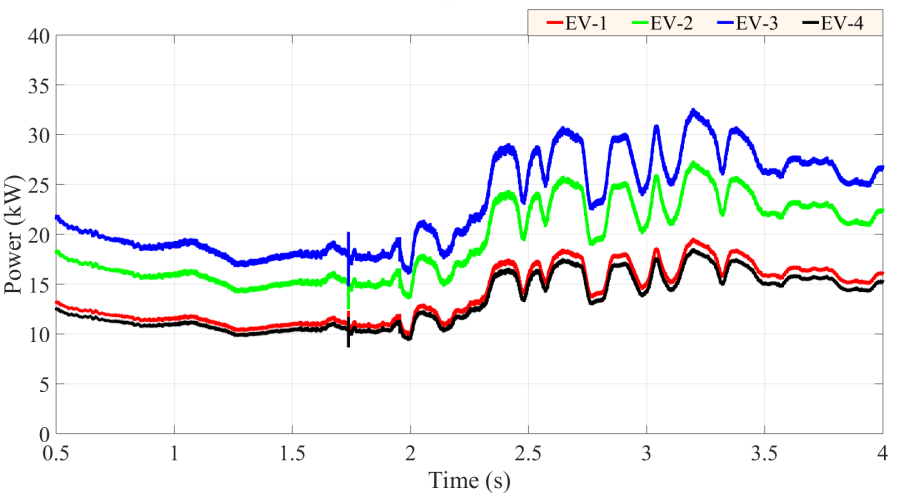

(e)

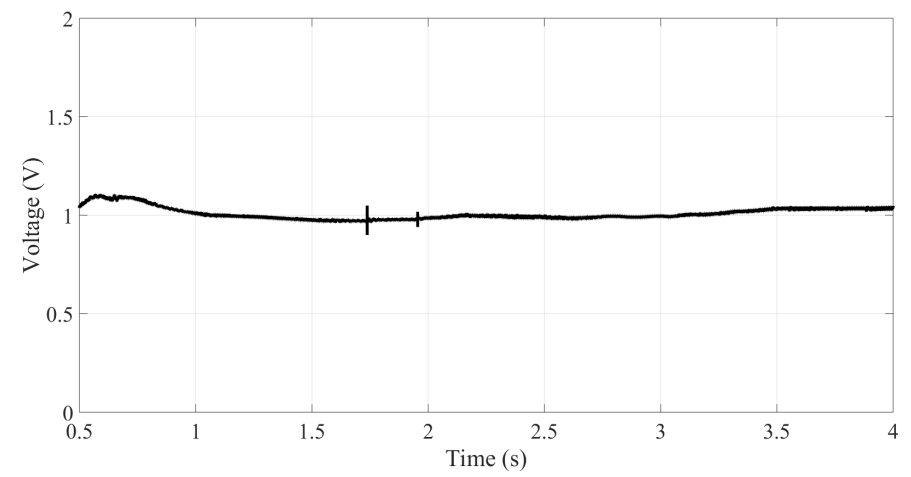

(b)

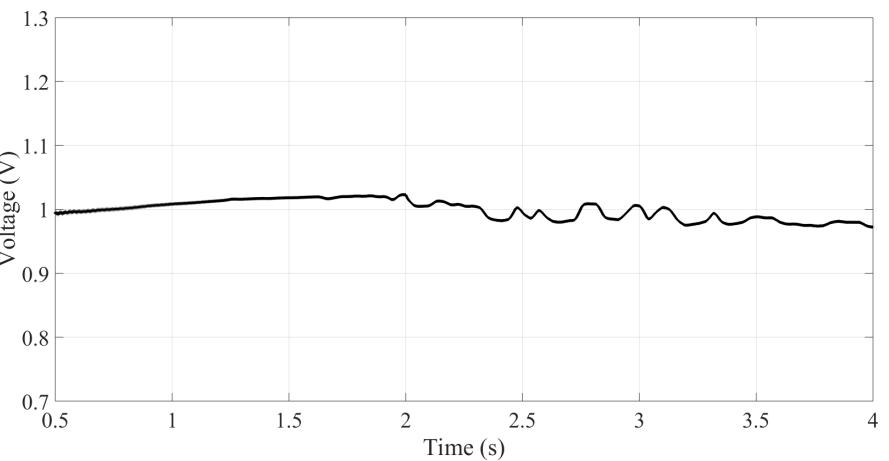

(d)

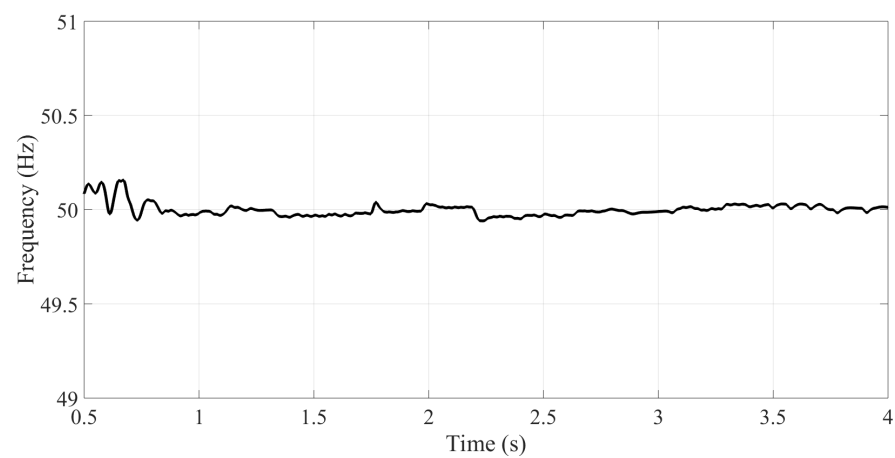

(f)

Fig. 5: Performance evaluation under variable solar insolation and commercial load profile. (a) Optimal incremental cost in c/kW. (b) AC bus voltage in per unit (p. u.). (c) Dynamic cost of all EV storages. (d) Normalized DC bus voltage. (e) Output power of all EV storages. (f) Frequency of the system.

However, an intentional islanding or grid reconnection have certain effects on the electrical parameters of the concerned microgrid. For this case study, at $\mathrm{t}=2.5 \mathrm{~s}$ the islanded hybrid AC/DC microgrid is reconnected with the grid. During the islanded operational mode, the EV storages provide ancillary V2M services by power sharing and voltage regulation to ensure stable microgrid operation. However, if the microgrid is reconnected with a stiff grid then further V2M operation might not be essential. As a result, EV storages are typically expected to be in charging mode during grid-tied microgrid operation. Furthermore, due to the support of the grid, the operating cost or incentives required for $\mathrm{V} 2 \mathrm{M}$ operation are reduced. This phenomenon can be observed in Figs. 6a and 6c, which represent the optimal incremental cost and the operating cost of all EV storages. It can be observed from both Figs. $6 \mathrm{a}$ and $6 \mathrm{c}$ that the overall cost is steeply reduced due to the presence of a stiff grid. Therefore, all EV storages enter into charging modes also known as grid-to-vehicle $(\mathrm{G} 2 \mathrm{~V})$ mode, for most of the period after $t=2.5 \mathrm{~s}$. The negative values of the cost function in Fig. $6 \mathrm{c}$ represents the dynamic cost required to charge a vehicle. The output charging or discharging power for all EV storages are shown in Fig. 6e. The electrical parameters such as the AC bus voltage, the DC bus voltage and the system frequency for this case are shown in Figs. $6 \mathrm{~b}, 6 \mathrm{~d}$ and $6 \mathrm{f}$ respectively. Due to grid reconnection, the AC bus voltage and the frequency quickly settle down by tracking the gridenforced reference values. The DC bus voltage exhibits a dip during the reconnection but quickly settles down due to the efficacy of the designed ODC for EV storage. 


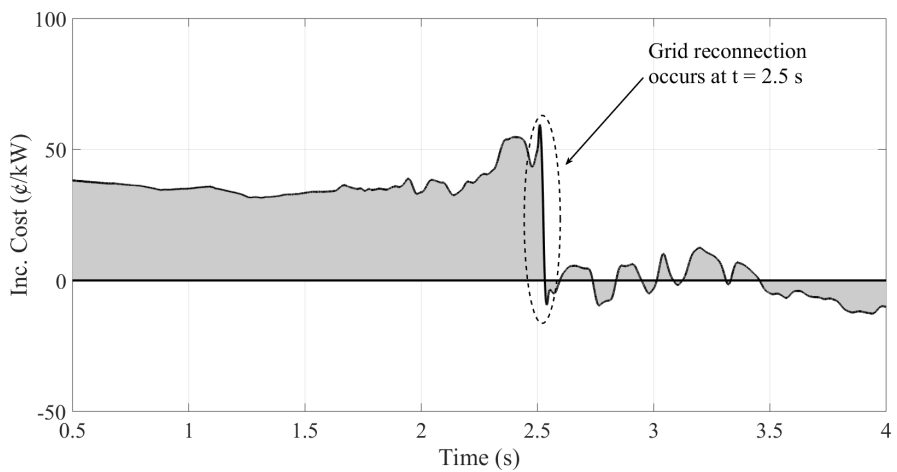

(a)

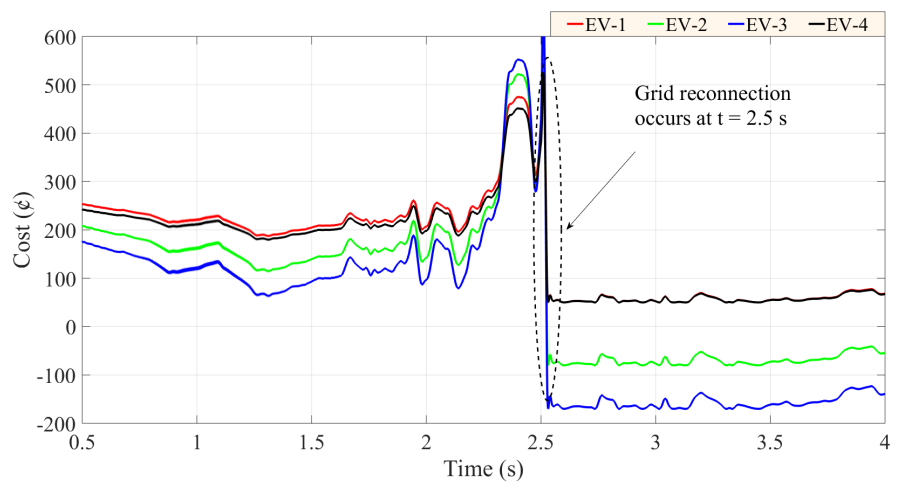

(c)

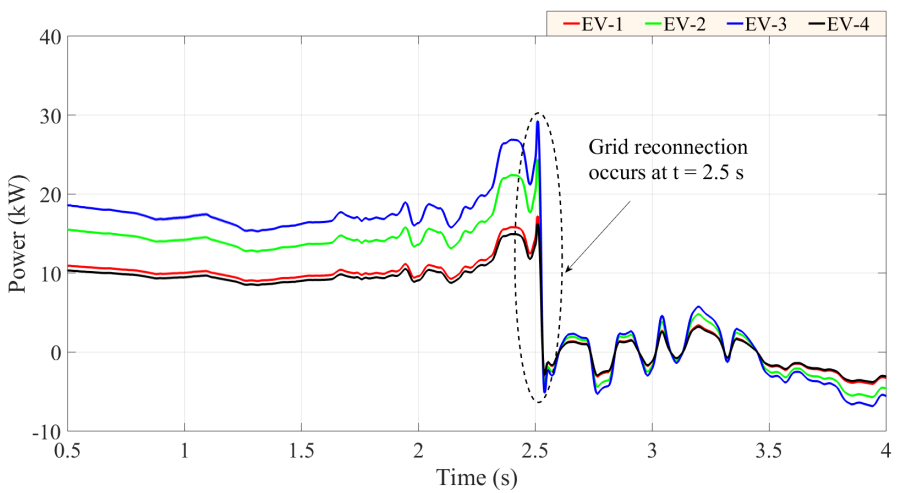

(e)

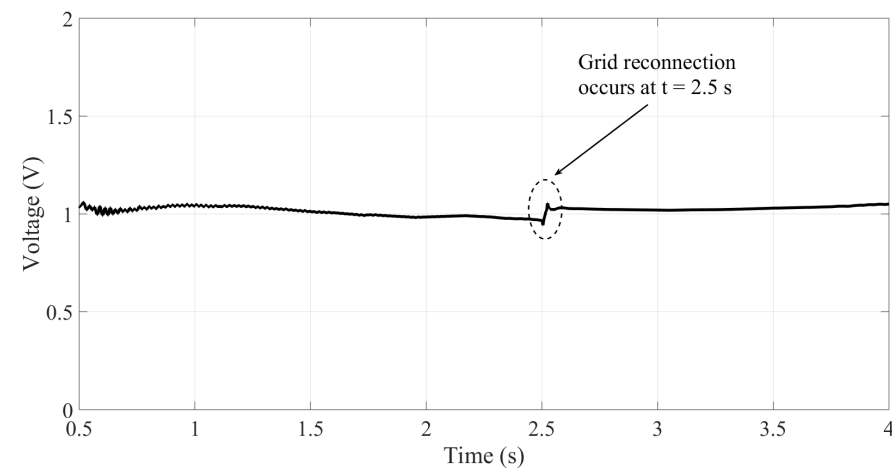

(b)

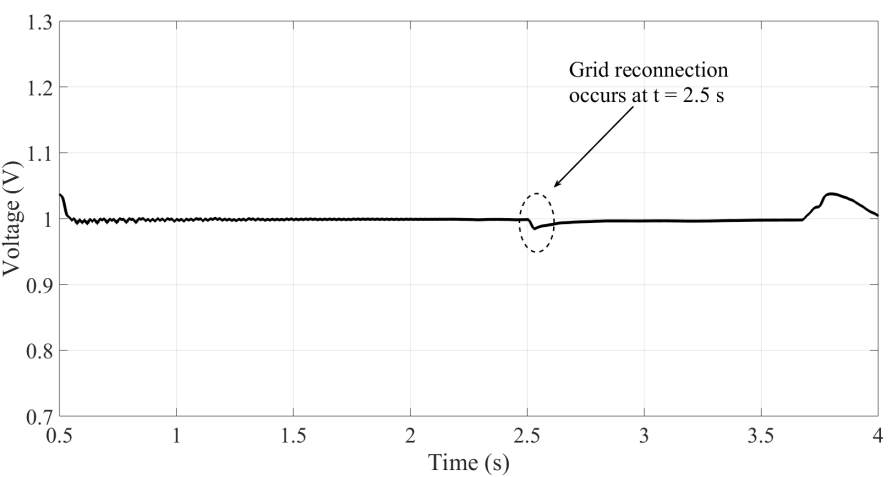

(d)

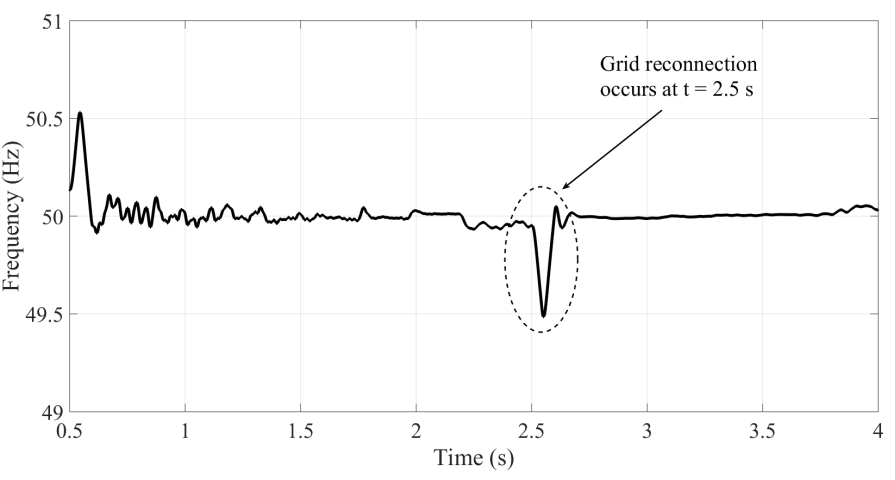

(f)

Fig. 6: Performance evaluation during and subsequent to grid reconnection. (a) Optimal incremental cost in c/kW. (b) AC bus voltage in per unit (p. u.). (c) Dynamic cost of all EV storages. (d) Normalized DC bus voltage. (e) Output power of all EV storages. (f) Frequency of the system.

\section{Case C: Effects of EV Disconnection and Time Delay}

Case $\mathrm{C}$ is dedicated to analyzing the effects of intentional EV service disruption on the system. As EV storages are individually owned, the designed V2M network needs to withstand sudden EV disconnection based on owners' desires. The designed framework should be robust enough to endure dynamic situations where EV owners possess the flexibility of including or excluding EV storages for V2M operations. The load profiles and the solar insolation used in the previous case are kept similar for this case study. At $\mathrm{t}=2 \mathrm{~s}$, EV-4 is disconnected from the system, emulating a fault at the V2M terminal or a service disruption condition. Figs. 7 and 8 present the dynamic operating cost and the output power profile for all EV storages before and after the disconnection of EV-4. As there is a shortage of supply due to this disconnection, other
EV storages need to cover this additional power requirement to make the system stable. As a result, the output powers of all EV storages have increased after $\mathrm{t}=2 \mathrm{~s}$. However, active EV storages require higher incentives to supply this additional discharged power. Therefore, it can be observed from Fig. 7 that there is an escalation in the operating cost profile after $\mathrm{t}$ $=2 \mathrm{~s}$.

Fig. 9 represents the results for the scenario when consecutively two EV storages are disconnected within a short interval. At $\mathrm{t}=1.5 \mathrm{~s} \mathrm{EV}-1$ is disconnected; as a result, other EV storages have increased their output power to meet the demand as expected. However, at $\mathrm{t}=3 \mathrm{~s}$ another EV storage (i.e., EV3 ) is disconnected. The microgrid system is now incapable of serving the load requirements due to the loss of two storages. As a result, the grid reconnection is initiated at $t=3 \mathrm{~s}$. The operating EV-2 and EV-4 power then become supplemented 


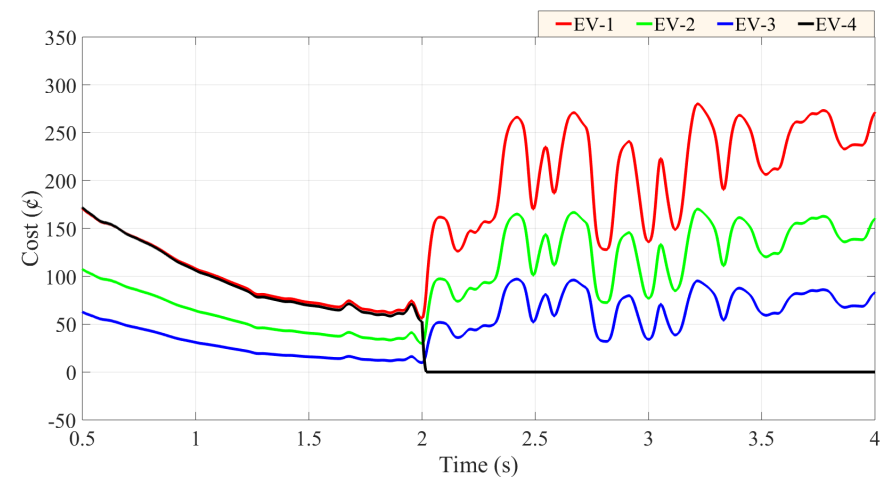

Fig. 7: Costs of all EV storages when EV-4 is intentionally disconnected at $t$ $=2 \mathrm{~s}$.

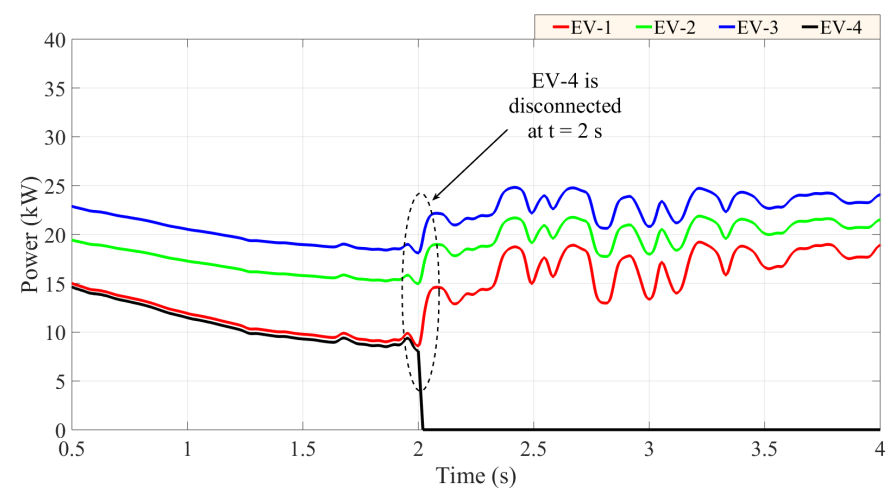

Fig. 8: Output powers of all EV storages when EV-4 is intentionally disconnected at $\mathrm{t}=2 \mathrm{~s}$.

by the grid to meet the load requirements.

The effects of a time delay on the power profiles of EV storages are presented in Fig. 10. A time delay of $30 \mathrm{~ms}$ is introduced at the distributed power-control loop. It can be observed from Fig. 10a that the outputs of the distributed controller for all EV storages, i.e. $u_{p}^{1}, u_{p}^{2}, u_{p}^{3}$ and $u_{p}^{4}$, converge to an average value of output power with time delay $T_{d}=0$ $\mathrm{ms}$. Conversely, introducing a delay of $30 \mathrm{~ms}$ deteriorates the controller performance on convergence. However, the response is stable, as $T_{d}$ is within the specified limit given in (8). As the output powers of all EV storages are driven by the values $u_{p}^{n}$, the discharged output powers from all EV storages are also affected by the time delay as shown in Fig. 10.

\section{CONCLUSiON}

A novel V2M control framework combining the centralized economic dispatch problem with the distributed EV coordination strategy is presented for optimal power-sharing and voltage regulation for a commercial neighborhood to operate as an autonomous electric entity. An aggregator model is developed that generates reference powers for all EV storages utilizing a centralized optimization technique to solve an economic dispatch problem. An EV storage controller is developed that tracks the reference power generated by the EV aggregator and can operate in either decentralized or distributed mode. Finally, a modified interlinking converter is designed that ensures proper power transportation between the AC and DC subgrids of a hybrid AC/DC microgrid intended for a commercial

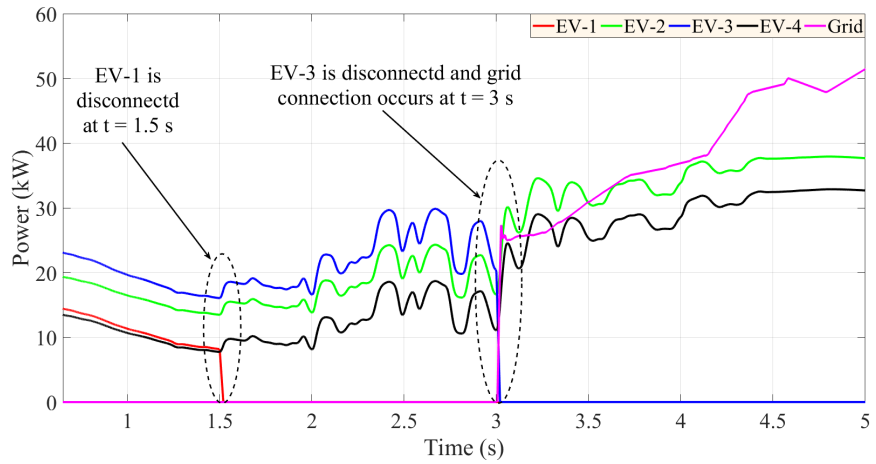

Fig. 9: Output powers of all EV storages when EV-1 is intentionally disconnected at $\mathrm{t}=1.5 \mathrm{~s}$, then EV-3 is disconnected at $\mathrm{t}=3 \mathrm{~s}$. The loss of two EV storages initiates grid reconnection at $\mathrm{t}=3 \mathrm{~s}$.
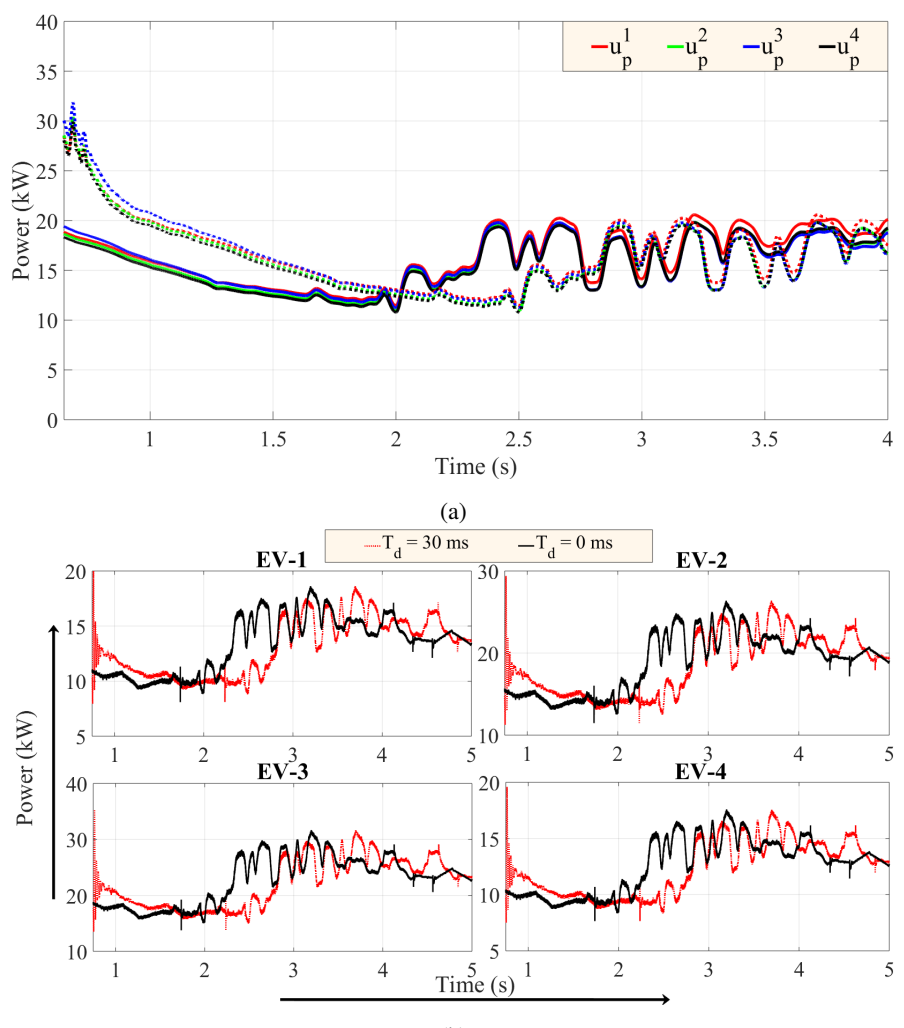

(b)

Fig. 10: Effects of $30 \mathrm{~ms}$ time delay on the system power profile. (a) Output $u_{p}^{n}$ of the distributed controllers for all EV storages with time delay (dotted) and without time delay (solid). (b) Discharged power of all EV storages with time delay (red) and without time delay (black).

locality. The developed control framework provides visibility on how the centralized aggregator model will interact with individual EV storage controllers. The proposed structure will offer not only technical efficacy concerning voltage/frequency regulation, and system stability but also provide economic benefits to EV owners and aggregators. Rigorous case studies conducted considering various scenarios such as generationload variability, intentional EV disconnection, time delay, and grid reconnection solidifies the effectiveness of the overall microgrid system. The future directive of this research is to incorporate additional functionalities such as improved power quality, reduced circulating current [36], [37] and robust 
performance during unbalanced weak grid scenario into the proposed V2M framework.

\section{REFERENCES}

[1] D. L. Gerber, V. Vossos, W. Feng, C. Marnay, B. Nordman, and R. Brown, "A simulation-based efficiency comparison of ac and dc power distribution networks in commercial buildings," Applied Energy, vol. 210, pp. $1167-1187,2018$.

[2] X. Liu, P. Wang, and P. C. Loh, "A hybrid AC/DC microgrid and its coordination control," IEEE Trans. Smart Grid, vol. 2, no. 2, pp. 278 286, June 2011.

[3] P. C. Loh, D. Li, Y. K. Chai, and F. Blaabjerg, "Autonomous control of interlinking converter with energy storage in hybrid AC/DC microgrid," IEEE Trans. Ind. Appl., vol. 49, no. 3, pp. 1374-1382, May 2013.

[4] N. Eghtedarpour and E. Farjah, "Power control and management in a hybrid AC/DC microgrid," IEEE Trans. Smart Grid, vol. 5, no. 3, pp. 1494-1505, May 2014.

[5] P. Wang, L. Goel, X. Liu, and F. H. Choo, "Harmonizing AC and DC: A hybrid AC/DC future grid solution," IEEE Power Energy Mag., vol. 11, no. 3, pp. 76-83, May 2013.

[6] Y. J. Kim, D. H. Blum, N. Xu, L. Su, and L. K. Norford, "Technologies and magnitude of ancillary services provided by commercial buildings," Proc. IEEE, vol. 104, no. 4, pp. 758-779, April 2016.

[7] M. S. Rahman, M. J. Hossain, and J. Lu, "Coordinated control of three-phase AC and DC type EV-ESSs for efficient hybrid microgrid operations," Energy Conversion and Management, vol. 122, pp. $488-$ 503, 2016

[8] M. S. Rahman, M. J. Hossain, J. Lu, and H. R. Pota, "A needbased distributed coordination strategy for ev storages in a commercial hybrid ac/dc microgrid with an improved interlinking converter control topology," IEEE Trans. Energy Convers., vol. 33, no. 3, pp. 1372-1383, Sep. 2018.

[9] S. Beer, T. Gomez, D. Dallinger, I. Momber, C. Marnay, M. Stadler, and J. Lai, "An economic analysis of used electric vehicle batteries integrated into commercial building microgrids," IEEE Trans. Smart Grid, vol. 3, no. 1, pp. 517-525, March 2012.

[10] G. Byeon, T. Yoon, S. Oh, and G. Jang, "Energy management strategy of the dc distribution system in buildings using the ev service model," IEEE Trans. Power Electron., vol. 28, no. 4, pp. 1544-1554, April 2013.

[11] G. Zhang, T. Tan, and G. Wang, "Real-time smart charging of electric vehicles for demand charge reduction at non-residential sites," IEEE Trans. Smart Grid, vol. PP, no. 99, pp. 1-1, 2017.

[12] Q. Yan, B. Zhang, and M. Kezunovic, "Optimized operational cost reduction for an ev charging station integrated with battery energy storage and pv generation," IEEE Trans. Smart Grid, vol. PP, no. 99, pp. 1-1, 2018.

[13] A. Bidram, A. Davoudi, F. L. Lewis, and J. M. Guerrero, "Distributed cooperative secondary control of microgrids using feedback linearization," IEEE Trans. Power Syst., vol. 28, no. 3, pp. 3462-3470, August 2013.

[14] A. Bidram, F. L. Lewis, and A. Davoudi, "Distributed control systems for small-scale power networks: Using multiagent cooperative control theory," IEEE Control Syst. Mag., vol. 34, no. 6, pp. 56-77, December 2014.

[15] V. Nasirian, S. Moayedi, A. Davoudi, and F. L. Lewis, "Distributed cooperative control of DC microgrids," IEEE Trans. Power Electron., vol. 30, no. 4, pp. 2288-2303, April 2015.

[16] Q. Shafiee, T. Dragicevic, J. C. Vasquez, and J. M. Guerrero, "Hierarchical control for multiple DC-microgrids clusters," IEEE Trans. Energy Convers., vol. 29, no. 4, pp. 922-933, December 2014.

[17] S. Moayedi and A. Davoudi, "Distributed tertiary control of DC microgrid clusters," IEEE Trans. Power Electron., vol. 31, no. 2, pp. 17171733, February 2016.

[18] P. Wang, C. Jin, D. Zhu, Y. Tang, P. C. Loh, and F. H. Choo, "Distributed control for autonomous operation of a three-port ac/dc/ds hybrid microgrid," IEEE Trans.s on Ind. Electron., vol. 62, no. 2, pp. 1279-1290, Feb 2015.

[19] T. Morstyn, B. Hredzak, and V. G. Agelidis, "Distributed cooperative control of microgrid storage," IEEE Trans. Power Syst., vol. 30, no. 5, pp. 2780-2789, September 2015 .

[20] T. Morstyn, A. Savkin, B. Hredzak, and V. Agelidis, "Multi-agent sliding mode control for state of charge balancing between battery energy storage systems distributed in a DC microgrid," IEEE Trans. Smart Grid, vol. PP, no. 99, pp. 1-1, 2017.
[21] M. S. Golsorkhi, Q. Shafiee, D. Lu, and J. M. Guerrero, "A distributed control framework for integrated photovoltaic-battery based islanded microgrids," IEEE Trans. Smart Grid, vol. PP, no. 99, pp. 1-1, 2016.

[22] M. Zeraati, M. E. H. Golshan, and J. Guerrero, "Distributed control of battery energy storage systems for voltage regulation in distribution networks with high PV penetration," IEEE Trans. Smart Grid, vol. PP, no. 99, pp. 1-1, 2016.

[23] N. Mahmud, A. Zahedi, and A. Mahmud, "A cooperative operation of novel PV inverter control scheme and storage energy management system based on ANFIS for voltage regulation of grid-tied PV system," IEEE Trans. Ind. Informat., vol. PP, no. 99, pp. 1-1, 2017.

[24] S. Moayedi and A. Davoudi, "Unifying distributed dynamic optimization and control of islanded dc microgrids," IEEE Trans. Power Electron., vol. 32, no. 3, pp. 2329-2346, March 2017.

[25] Z. Wang, F. Liu, Y. Chen, S. H. Low, and S. Mei, "Unified distributed control of stand-alone de microgrids," IEEE Trans. Smart Grid, vol. PP, no. 99, pp. 1-1, 2017.

[26] T. L. Vandoorn, B. Meersman, J. D. M. D. Kooning, and L. Vandevelde, "Analogy between conventional grid control and islanded microgrid control based on a global DC-link voltage droop," IEEE Trans. Power Del., vol. 27, no. 3, pp. 1405-1414, July 2012.

[27] — , "Transition from islanded to grid-connected mode of microgrids with voltage-based droop control," IEEE Trans. Power Syst., vol. 28 , no. 3, pp. 2545-2553, August 2013.

[28] Z. Wang, W. Wu, and B. Zhang, "A distributed control method with minimum generation cost for dc microgrids," IEEE Trans. Energy Convers., vol. 31, no. 4, pp. 1462-1470, Dec 2016.

[29] H. Han, H. Wang, Y. Sun, J. Yang, and Z. Liu, "Distributed control scheme on cost optimisation under communication delays for dc microgrids," IET Generation, Transmission Distribution, vol. 11, no. 17, pp. 4193-4201, 2017.

[30] G. Binetti, A. Davoudi, F. L. Lewis, D. Naso, and B. Turchiano, "Distributed consensus-based economic dispatch with transmission losses," IEEE Trans. Power Syst., vol. 29, no. 4, pp. 1711-1720, July 2014.

[31] Lu Jiaxin, Zhang Yingchao, Qian Xisen, Long Jiangtao, and Zhao Zhengming, "A novel virtual impedance method for droop controlled parallel ups inverters with wireless control," in 2014 IEEE Conference and Expo Transportation Electrification Asia-Pacific (ITEC AsiaPacific), Aug 2014, pp. 1-5.

[32] J. Hetzer, D. C. Yu, and K. Bhattarai, "An economic dispatch model incorporating wind power," IEEE Transactions on Energy Conversion, vol. 23, no. 2, pp. 603-611, June 2008.

[33] R. Olfati-Saber, J. A. Fax, and R. M. Murray, "Consensus and cooperation in networked multi-agent systems," Proc. IEEE, vol. 95, no. 1, pp. 215-233, January 2007

[34] R. Olfati-Saber and R. M. Murray, "Consensus problems in networks of agents with switching topology and time-delays," IEEE Trans. Autom. Control, vol. 49, no. 9, pp. 1520-1533, September 2004.

[35] J. M. Guerrero, P. C. Loh, T. L. Lee, and M. Chandorkar, "Advanced control architectures for intelligent microgrids - part II: Power quality, energy storage, and AC/DC microgrids," IEEE Trans. Ind. Electron., vol. 60, no. 4, pp. 1263-1270, April 2013.

[36] Y. Xia, Y. Peng, P. Yang, M. Yu, and W. Wei, "Distributed coordination control for multiple bidirectional power converters in a hybrid ac/dc microgrid," IEEE Transactions on Power Electronics, vol. 32, no. 6, pp. 4949-4959, June 2017.

[37] Y. Xia, W. Wei, M. Yu, X. Wang, and Y. Peng, "Power management for a hybrid ac/dc microgrid with multiple subgrids," IEEE Transactions on Power Electronics, vol. 33, no. 4, pp. 3520-3533, April 2018. 


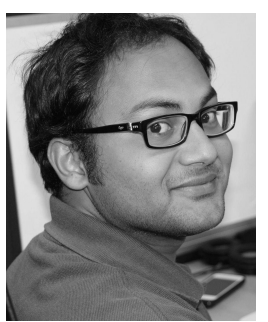

Md Shamiur Rahman (S'14) received the B.Sc. in electrical and electronic engineering from Islamic University of Technology (IUT), Bangladesh in 2011 and the Ph.D. degree from the Macquarie University, Australia in 2018. He is currently working as a power system planning engineer in ElectraNet Pty. Ltd, Adelaide, Australia.

Prior to starting Ph.D. studies, he worked as a radio frequency (RF) engineer in Huawei Technologies Bangladesh Limited from 2011 to 2013. Later, from 2013 to 2014 he worked as a core and IP network planning (CNIP) engineer in Robi Axiata Limited. His research interests include microgrid, distributed control techniques, power management, vehicleto-grid (V2G), energy storage, renewable energy and distributed generation.

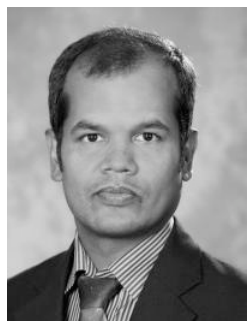

M. Jahangir Hossain (M'10-SM'13) received the B.Sc. and M.Sc. Eng. degrees from Rajshahi University of Engineering and Technology (RUET), Bangladesh, in 2001 and 2005, respectively, and the $\mathrm{Ph} . \mathrm{D}$. degree from the University of New South Wales, Australia, all in electrical and electronic engineering.

$\mathrm{He}$ is currently an Associate Professor with the Department of Engineering, Macquarie University. Before joining there, he served as a Senior Lecture and a Lecturer in the Griffith School of Engineering, Griffith University, for five years and as a Research Fellow in the School of Information Technology and Electrical Engineering, University of Queensland, Brisbane, Australia. His research interests include renewable energy integration and stabilization, voltage stability, micro grids and smart grids, robust control, electric vehicles, flexible ac transmission systems devices, and energy storage systems.

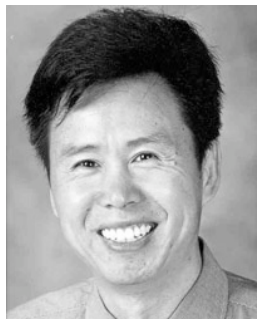

Junwei Lu (M'95-SM'05) received the degree in electrical engineering from Xian Jiaotong University, $\mathrm{Xi}$ 'an, China, the M.Eng. degree in electronic and computer engineering from the National Toyama University, Toyama, Japan, and the Ph.D. degree in electrical and computer engineering from the National Kanazawa University, Kanazawa, Japan, in 1991.

From 1976 to 1984, he worked with the electrical power industry (now called State Grid) in China, where he was involved in the various national research projects including a $500 \mathrm{kV}$ power system for electrical power industry In 1985, his academic study and research was in the area of computational electromagnetics at the Laboratory of Electrical Communication Engineering at Toyama University, Toyama Prefecture, Japan. In 1988, he worked on the applied computational electromagnetics and was involved in the development of magnetics devices and electric machines with the Laboratory of Electrical Energy Conversion, Kanazawa University. He joined the School of Microelectronic Engineering, mainly working on power electronics and electric machines, Griffith University, Brisbane, Australia, in 1992, and moved to the Gold Coast campus to establish Electrical and Electronic Engineering as a Foundation Professor in 2011. He has published more than 250 journal and conference papers and three coauthored books in the area of electromagnetic compatibility (EMC) V2G and harmonic balance finite element method, and holds more than 10 international patents related to smart antennas and high frequency transformers. His fields of interest are computational electromagnetics, EMC computer modeling and simulation, radio frequency/microwave devices and circuits, and high-frequency magnetics for power electronics, and renewable energy system. His current research interests include smart transformer and V2G with a built-in statcom inverter and smart hybrid AC/DC microgrid.

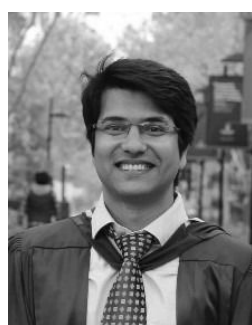

Fida Hasan Md Rafi (S'14) received his B.Sc. degree in electrical and electronic engineering from Khulna University of Engineering and Technology, Bangladesh, in 2012, and the Ph.D. degree from the Macquarie University, Sydney, Australia in 2017. $\mathrm{He}$ is currently working as a network development engineer at Electranet, Adelaide. Prior to this position, he was working as a post-doctoral research fellow at Griffith University, Australia. His current research interests include smart inverter, power system dynamic analysis, renewable energy system, unbalanced network analysis, STATCOM, energy storage system and electric vehicles.

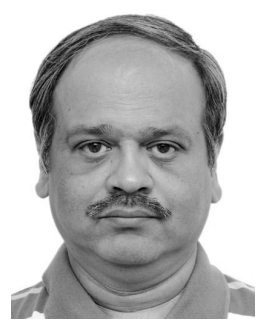

Sukumar Mishra (M'97-SM'04) is a Professor at Indian Institute of Technology Delhi and has been part of the institute for the past 15 years. He has published over 200 research articles (including papers in international journals, conferences and book chapters).

Prof. Mishra has won many accolades throughout his academic tenure of 25 years. He has been a recipient of INSA medal for young scientist (2002), INAE young engineer award (2009), INAE silver jubilee young engineer award (2012) and has recently won the Samanta Chandra Shekhar Award (2016). He has been granted fellowship from many prestigious technical societies like IET (UK), NASI (India), INAE (India), IETE (India) and IE (India) and is also recognized as the INAE Industry Academic Distinguish Professor. Currently, Prof. Mishra is holding the position of Vice Chair of Intelligent System Subcommittee of Power and Energy society (PES) of IEEE. Apart from all research and academic collaborations, Prof. Mishra is very actively involved in industrial collaborations. Prof. Mishra is currently acting as INAE Chair professor and has previously delegated as the NTPC and Power Grid Chair professor. $\mathrm{He}$ is also serving as an Independent Director of the Cross Border Power Transmission Company Ltd. and the River Engineering Pvt. Ltd.

Prof. Mishra's research expertise lies in the field of Power Systems, Power Quality Studies, Renewable Energy and Smart Grid. Prof. Mishra is currently serving as an Editor for the IEEE Transactions on Sustainable Energy, IEEE Transactions on Smart Grid and an Associate Editor for the IET Generation, Transmission \& Distribution journal. 